\title{
Pure subgame-perfect equilibria in free transition games
}

Citation for published version (APA):

Kuipers, J., Flesch, J., Schoenmakers, G. M., \& Vrieze, K. (2008). Pure subgame-perfect equilibria in free transition games. METEOR, Maastricht University School of Business and Economics. METEOR Research Memorandum No. 027 https://doi.org/10.26481/umamet.2008027

Document status and date:

Published: 01/01/2008

DOI:

10.26481/umamet.2008027

Document Version:

Publisher's PDF, also known as Version of record

\section{Please check the document version of this publication:}

- A submitted manuscript is the version of the article upon submission and before peer-review. There can be important differences between the submitted version and the official published version of record.

People interested in the research are advised to contact the author for the final version of the publication, or visit the DOI to the publisher's website.

- The final author version and the galley proof are versions of the publication after peer review.

- The final published version features the final layout of the paper including the volume, issue and page numbers.

Link to publication

\footnotetext{
General rights rights.

- You may freely distribute the URL identifying the publication in the public portal. please follow below link for the End User Agreement:

www.umlib.nl/taverne-license

Take down policy

If you believe that this document breaches copyright please contact us at:

repository@maastrichtuniversity.nl

providing details and we will investigate your claim.
}

Copyright and moral rights for the publications made accessible in the public portal are retained by the authors and/or other copyright owners and it is a condition of accessing publications that users recognise and abide by the legal requirements associated with these

- Users may download and print one copy of any publication from the public portal for the purpose of private study or research.

- You may not further distribute the material or use it for any profit-making activity or commercial gain

If the publication is distributed under the terms of Article $25 \mathrm{fa}$ of the Dutch Copyright Act, indicated by the "Taverne" license above, 
Jeroen Kuipers, Janos Flesch, Gijs Schoenmakers, Koos Vrieze

Pure Subgame-Perfect Equilibria in Free Transition Games

$\mathrm{RM} / 08 / 027$

JEL code: C73

\section{METE@R}

Maastricht research school of Economics of TEchnology and ORganizations

Universiteit Maastricht

Faculty of Economics and Business Administration P.O. Box 616

NL - 6200 MD Maastricht

phone : ++31433883830

fax : $\quad$ ++31433884873 


\title{
Pure Subgame-Perfect Equilibria in Free Transition Games
}

\author{
J. Kuipers*† J. Flesch $;$ G. Schoenmakers*, K. Vrieze*
}

August 22, 2008

\begin{abstract}
We consider a class of stochastic games, where each state is identified with a player. At any moment during play, one of the players is called active. The active player can terminate the game, or he can announce any player, who then becomes the active player. There is a non-negative payoff for each player upon termination of the game, which depends only on the player who decided to terminate. We give a combinatorial proof of the existence of subgame-perfect equilibria in pure strategies for the games in our class.
\end{abstract}

\section{Introduction}

We consider a class of stochastic games with perfect information and deterministic transitions. Only upon termination the players receive a payoff, which depends only on the player who terminated. Available results in literature do not tell whether subgame-perfect equilibria exist for the games in our class. In this article, we provide an explicit description of a subgame-perfect equilibrium in pure strategies in two simple rules of play.

Proving the existence of subgame-perfect equilibria for undiscounted stochastic games is generally considered a tough problem. Due to a result by Liggett and Lippman (1969), stationary subgame-perfect equilibria in pure strategies are known to exist for 2-player zero-sum games with perfect information. Thuijsman and Raghavan (1996) use Liggett and Lippman's result to prove the existence of pure Nash-equilibria for $n$-player games with perfect information. These equilibria are not subgame-perfect, since they involve threats where $n-1$ players must cooperate to minimize a deviating player, without regard to their own payoff. Solan and Vieille (2003) and Solan (2005) prove the existence of subgameperfect $\epsilon$-equilibria for two classes of $n$-player perfect information games, where, like in our class, payoffs are at termination and are determined by the terminating player. The main difference with our class is that the transitions for these two classes are not influenced by the players. Solan and Vieille (2003) consider a class where the order of active players is predetermined, and Solan (2005) considers a class where each player becomes active with probability $\frac{1}{n}$. Also in contrast to these papers, this article uses purely combinatorial

\footnotetext{
*MICC-Mathematics, Maastricht University, P.O. Box 616, 6200MD, Maastricht, The Netherlands.

${ }^{\dagger}$ Corresponding author: e-mail: kuipers@micc.unimaas.nl

${ }^{\ddagger}$ Dept. of Quantitative Economics, Maastricht University, P.O. Box 616, 6200MD, Maastricht, The Netherlands.
} 
arguments. The proof is rather specialized to the class under consideration, but may initiate further results in this area.

For a certain class of coalition formation games with deterministic transitions and payoffs at termination, Bloch (1996) claims the existence of subgame-perfect equilibria. His approach is to approximate the undiscounted games with discounted ones. Due to a result by Fudenberg and Levine (1983), the existence of pure subgame-perfect equilibria for the discounted games is guaranteed, so it is a matter of showing that the limit of subgame-perfect discounted equilibria is a subgame-perfect undiscounted equilibrium. This approach seems feasible for our class of games as well, given the similarities between the two classes. In his proof, Bloch claims the existence of an infinite subsequence of the discounted subgameperfect equilibria that is constant. Bloch deduces this from the fact that there are a finite number of subgame-perfect equilibria outcomes, but we believe this reasoning is incorrect. Even if it were possible to complete Bloch's approach, it would not reveal much of the structure of the subgame-perfect equilibria. This is in contrast to our approach, which allows the easy computation of a subgame-perfect equilibrium. The results in this article do not easily extend to the class of games considered by Bloch.

In Section 2, we introduce our game formally, and we show by example that a subgameperfect equilibrium may not exist among stationary strategies. In Section 3, we introduce the set of persistent players that can appear as the outcome of subgame-perfect equilibrium play. We provide intuitive motivations for the definitions, and we derive the relevant mathematical properties of that set. Section 4 contains the description of subgame-perfect equilibrium strategies in two rules of play, one rule for persistent players and another rule for non-persistent players.

\section{Free Transition Games and Illustrative Example}

Let $N$ be a finite set of players. A payoff vector is associated with each player $n \in N$, denoted by $v(n) \in \mathbb{R}^{N}$, and which satisfies $v(n) \geq 0$. At any moment during play, one of the players is called active. The active player, say $n$, always has the option to terminate the game. In this case, players receive payoffs according to $v(n)$. The active player can also announce any player (including himself), who then becomes the new active player. If a new active player is announced there is no payoff. The stochastic game defined in this way is called a free transition game, as the model places no restrictions on the choice of an active player.

A history of the free transition game is a finite sequence of players. It can be interpreted as the list of active players during play in chronological order. The first player in the history of the game is the initial player, and the last player represents the active player.

For $n \in N$, let $H^{n}$ denote the set of all possible histories ending with $n$. Let $\Delta$ denote the set of all probability vectors over $N \cup$ \{quit\}. A strategy for $n \in N$ is a function $\sigma^{n}: H^{n} \rightarrow \Delta$. It can be interpreted as a prescription for play for player $n$ for every possible situation (history) he might face. The strategy is pure if, for every $h \in H^{n}, \sigma^{n}(h)$ prescribes one of the actions in $N \cup$ \{quit\} with probability 1. The strategy is stationary if $\sigma^{n}(h)=\sigma^{n}\left(h^{\prime}\right)$ for all $h, h^{\prime} \in H^{n}$. Joint strategies $\left(\sigma^{n}\right)_{n \in N}$ are a strategy profile. The strategy profile is called pure / stationary if $\sigma^{n}$ is pure / stationary for all $n \in N$. 
Let $n \in N$ denote the initial player of the game. For a strategy profile $\sigma$, let $E^{n}(\sigma) \in$ $\mathbb{R}^{N}$ denote the vector of expected outcomes (payoffs if the game terminates and zeros otherwise) when all players play according to $\sigma$. Notice that $E^{n}(\sigma)$ is well-defined, since for each $m \in N$, the probability of termination at $m$ is well-defined by $\sigma$. For a strategy profile $\sigma$ and a player $m \in N$, say that a strategy profile $\pi$ is an $m$-deviation from $\sigma$ if all players, except possibly $m$, have the same strategy in $\pi$ and $\sigma$. Notice that $\sigma$ is an $m$-deviation from $\sigma$ for every $m \in N$. A strategy profile $\sigma$ is a Nash-equilibrium if, for every initial player $n \in N$ and every $m \in N$, we have

$$
E^{n}(\sigma)_{m}=\max \left\{E^{n}(\pi)_{m} \mid \pi \text { is an } m \text {-deviation from } \sigma\right\} .
$$

For the games in our class a trivial pure stationary Nash-equilibrium is constructed as follows. The initial player is supposed to terminate always. All other players are supposed to announce the initial player always (if they ever get their turn). Notice that the initial player cannot improve himself by deviating, since the game will never terminate (which yields a payoff of 0 ) if he always refuses to terminate. The other players cannot improve by changing their strategy either, since the initial player will terminate the game before they get their turn.

For a strategy profile $\sigma$ and a history $h$ ending at initial player $n$, denote by $\sigma \mid h$ the strategy profile that prescribes the players to play according to $\sigma$, but as if history $h$ had preceded the actual history. The strategy profile $\sigma$ is a subgame-perfect equilibrium if, for every history $h, \sigma \mid h$ is a Nash-equilibrium.

Consider the following example with 3 players. Let $N=\{1,2,3\}$, and let payoff vectors be given by

$$
v(1)=\left(\begin{array}{l}
2 \\
1 \\
4
\end{array}\right), v(2)=\left(\begin{array}{l}
4 \\
2 \\
1
\end{array}\right), v(3)=\left(\begin{array}{l}
1 \\
4 \\
2
\end{array}\right) .
$$

Let us denote the earlier given construction of a Nash-equilibrium for this example by $\sigma$. We first show that $\sigma$ is not a subgame-perfect equilibrium. Let 1 be the initial player and let $h=(3,1)$. According to $\sigma \mid h$, players should act as if 3 is the initial player. Thus, according to $\sigma \mid h$, player 1 is supposed to announce 3 and player 3 will then terminate. Obviously $\sigma \mid h$ is not a Nash-equilibrium, since it is better for player 1 to quit.

We now show that the example admits no subgame-perfect equilibrium in stationary strategies. Obviously, for such an equilibrium, a player should announce himself with probability strictly smaller than 1 , as otherwise his payoff will be 0 if he becomes active. We can even assume that a player places probability 0 on announcing himself. If this probability is positive, say $p$, then it can be set to 0 , and the probabilities on the other three actions can be multiplied by $\frac{1}{1-p}$. This adaptation of the strategy does not change the expected outcome or the existence of profitable deviations. Further, for a subgame-perfect equilibrium, at least one of the players must place positive probability on quitting. Because of symmetry, we may assume that player 1 quits with positive probability, and we may even assume that this probability is at least as high as for any other player. Then it is straightforward to prove that the expected payoff for player 3 will be strictly higher than 2 if he announces 1 always. For a stationary equilibrium, it must then be true that player 3 does not quit. Subsequently, it follows that player 2 should quit with probability 1, as 
any other action would lead to a payoff of 1 with positive probability, and never to a payoff of 4 . Next we conclude that player 1 should never quit, since only this would give player 1 the maximal payoff 4 . This contradicts that we were allowed to assume a positive probability on quitting for player 1 .

Now consider the following way of playing the game. At the start of the game, a 'scapegoat' player is chosen. The active player is supposed to quit if he is the scapegoat or if quitting yields 1 for the scapegoat. Otherwise, the active player is supposed to announce the player where the scapegoat receives 1 . If the active player does not play according to this plan, he will become the new scapegoat. Since it is possible to uniquely determine the scapegoat for any preceding history $h$, the plan describes a pure but history dependent strategy profile.

If players follow the rule, then the game will terminate after at most one announcement. Following the rule yields at least 2 for the active player: He will receive 2 if the rule tells him to quit and he will receive 4 if the rule tells him to announce another player. If a player deviates from the rule, he will receive at most 2. This is trivial if a player quits when he is not supposed to do so. Any other deviation results in becoming the scapegoat, and this also yields at most 2. As this works for every possible preceding history, the plan constitutes a subgame-perfect equilibrium.

\section{The Persistent Set}

For two players $n, m \in N$, we say that $m$ punishes $n$ if $v(m)_{n} \leq v(n)_{n}$. Let $Q \subseteq N$. For $n \in N$ and $m \in N \backslash Q$, we say that $(n, m)$ is a safe step with respect to $Q$ if for all $q \in Q$ such that $q$ punishes $n, q$ punishes $m$. For $m \in Q$, we say that $(n, m)$ is a safe step with respect to $Q$ if the previous condition holds and if moreover $m$ does not punish $n$. Notice that $(n, n)$ is a safe step for all $n \notin Q$, and that $(n, n)$ is not a safe step for all $n \in Q$. It is straightforward to prove that safe steps are transitive, i.e. if $(n, m)$ and $(m, p)$ are safe steps with respect to $Q$, then $(n, p)$ is a safe step with respect to $Q$. Call $n \in Q$ persistent with respect to $Q$ if $(n, m)$ is not safe for every $m \in Q$. Denote the set of persistent players with respect to $Q$ by $P(Q) \subseteq Q$. Now define the sequence $\left(P^{k}\right)_{k \in \mathbb{N}}$ by $P^{0}=N$ and $P^{k}=P\left(P^{k-1}\right)$ for $k>0$. Notice that the sequence $\left(P^{k}\right)_{k \in \mathbb{N}}$ converges in a finite number of steps, since $P^{k+1} \subseteq P^{k}$ for all $k \geq 0$. We denote the limit by $P^{*}$. We call $P^{*}$ the persistent set, and we speak of a safe step, when the step is safe with respect to $P^{*}$.

To have an interpretation of the definitions, assume that play can be expected to terminate at a player in $Q$. Suppose that $n \in N$ is active and that $(n, m)$ is a safe step with respect to $Q$. Consider what happens if player $n$ decides to announce $m$ as the new active player. If player $m$ quits the game, which 'implies' that $m \in Q$, then player $n$ will not regret his decision to announce $m$, since $m$ does not punish $n$. If player $m$ continues the game, then we trust him to let play terminate at a player in $Q$ that does not punish $m$. The transitivity of safe steps ensures that such a player does not punish $n$ either. Therefore, also if $m$ continues the game, player $n$ will not regret his decision to announce $m$.

We interpret the set $P(Q)$ as the subset of $Q$, where termination will actually take place. Notice that for any $q \in Q \backslash P(Q)$, a safe step to another player in $Q$ is possible, by definition 
of $P(Q)$. After this step, not even a sequence of safe steps back to $q$ exists. Otherwise, it would follow by transitivity that also $(q, q)$ is safe, contradicting our earlier observation that $(q, q)$ is not safe for every $q \in Q$. Now, if we assume that none of the players will risk playing a step that is not safe with respect to $Q$, it follows that termination at $q$ cannot happen anymore once $q$ has played safe.

Now merely assume that the game will terminate. According to our interpretation of $P(Q)$, we can then expect the game to actually terminate in $P^{1}=P\left(P^{0}\right)$. We can repeat this reasoning and restrict further to $P^{2}, P^{3}, \ldots$, etcetera. Hence, once we assume that the game will terminate, our interpretations 'force' us to believe that it will actually terminate in $P^{*}$.

To further illustrate the definitions, consider the following example. Let $N=\{1,2,3,4\}$, and let payoff vectors be given by

$$
v(1)=\left(\begin{array}{l}
1 \\
1 \\
1 \\
3
\end{array}\right), v(2)=\left(\begin{array}{l}
1 \\
1 \\
1 \\
1
\end{array}\right), v(3)=\left(\begin{array}{l}
2 \\
1 \\
2 \\
1
\end{array}\right), v(4)=\left(\begin{array}{l}
1 \\
2 \\
1 \\
2
\end{array}\right)
$$

Notice that every player punishes 3 and that 3 does not punish 1 . Therefore, the step $(1,3)$ is safe with respect to $N$. This is also the only safe step with respect to $N$. Therefore, $P^{1}=\{2,3,4\}$. Now notice that every player in $\{2,3,4\}$ punishes 4 and that 4 does not punish 2 . Therefore, the step $(2,4)$ is safe with respect to $\{2,3,4\}$. The steps $(1,3)$ and $(2,4)$ are the only safe steps with respect to $\{2,3,4\}$. Therefore, $P^{2}=\{3,4\}$. It easily verified that the steps $(1,3)$ and $(2,4)$ are the only two safe steps with respect to $\{3,4\}$. Hence, $P^{3}=P^{2}=\{3,4\}$. This shows that the persistent set in this example is $P^{*}=\{3,4\}$, and that the safe steps are $(1,3)$ and $(2,4)$. Our interpretation of $P^{*}$ is supported here by the fact that the example has a stationary subgame-perfect equilibrium, where the two players of $P^{*}$ always quit, and where the other two players always play their only available safe step.

The relevant mathematical properties of $P(Q)$ are listed in the following proposition.

Proposition 1 Let $Q \subseteq N$ and $Q \neq \emptyset$. Then

(a) $P(Q) \neq \emptyset$.

(b) For every $q \in Q \backslash P(Q), p \in P(Q)$ exists such that $(q, p)$ is safe with respect to $Q$.

(c) For every $p \in P(Q)$ and every $q \in Q$, the step $(p, q)$ is not safe with respect to $Q$.

Proof. Notice that (c) is true by definition and that (a) is a corollary of (b). Therefore, we only prove (b). Let $q \in Q \backslash P(Q)$. Starting at $q$, construct a sequence of players in $Q$, such that each step in the sequence is safe with respect to $Q$. This sequence is continued as long as players are visited that are not persistent with respect to $Q$. We observed before that no sequence of safe steps in $Q$ returns to the same player. Hence, the sequence is without cycles. It is therefore finite and it terminates at $p \in P(Q)$. Transitivity implies that $(q, p)$ is safe with respect to $Q$.

The relevant mathematical properties of $P^{*}$ are in the following proposition. 


\section{Proposition 2}

(a) $P^{*} \neq \emptyset$.

(b) For every $n \notin P^{*}, p \in P^{*}$ exists such that $(n, p)$ is safe.

(c) For every $p \in P^{*}$ and every $n \in N$, the step $(p, n)$ is not safe.

Proof. Notice that (a) follows from repeated application of Proposition 1, part (a). To prove (b) and (c), it is sufficient to prove the following two claims for all $k \geq 1$.

1. For every $n \notin P^{k}, p \in P^{k}$ exists such that $(n, p)$ is safe with respect to $P^{k-1}$.

2. If $p \in P^{k}$ and $n \in N$, then $(p, n)$ is not safe with respect to $P^{k-1}$.

We prove claim (1) by induction. That the claim is true for $k=1$, follows directly from Proposition 1, claim (b), applied with $Q=N=P^{0}$. Now assume the claim is true for some $k>0$. To prove claim (1) for $k+1$, let $n \notin P^{k+1}$. If $n \in P^{k}$, then a safe step with respect to $P^{k}$ to a player in $P^{k+1}$ exists, by Proposition 1, claim (b), and we are done. If $n \notin P^{k}$, then by the induction hypothesis, $p \in P^{k}$ exists such that $(n, p)$ is a safe step with respect to $P^{k-1}$. This step is also safe with respect to $P^{k}$, since $P^{k} \subseteq P^{k-1}$, so we are done immediately if $p \in P^{k+1}$. If $p \notin P^{k+1}$, then by Proposition 1 , claim (b), there is a safe step $\left(p, p^{\prime}\right)$ with respect to $P^{k}$, such that $p^{\prime} \in P^{k+1}$. By transitivity, $\left(n, p^{\prime}\right)$ is safe with respect to $P^{k}$.

We prove claim (2) by contradiction. Let $p \in P^{k}$ and $n \in N$, and suppose that $(p, n)$ is a safe step with respect to $P^{k-1}$. If $n \in P^{k-1}$, then we obtain an immediate contradiction with Proposition 1, claim (c). If $n \notin P^{k-1}$ (which implies $k \geq 2$ ), then $p^{\prime} \in P^{k-1}$ exists, such that $\left(n, p^{\prime}\right)$ is a safe step with respect to $P^{k-2}$, by claim (1) which we just proved. The step $\left(n, p^{\prime}\right)$ is also safe with respect to $P^{k-1}$, since $P^{k-1} \subseteq P^{k-2}$. By the transitivity

of safe steps, it now follows that $\left(p, p^{\prime}\right)$ is a safe step with respect to $P^{k-1}$. This contradicts with Proposition 1, claim (c).

\section{A Subgame-Perfect Equilibrium}

Under the rules that we present in this section, a non-persistent player is always required to play a safe step, and a persistent player is either supposed to terminate by himself, or to announce the (persistent) player who should terminate.

The rules keep track of a list of players who deviated by playing a step that is not safe. The players on this list are treated much like the scapegoat player of the example in Section 2. An active persistent player is supposed to terminate only if there is no persistent player who punishes one of the players on the list and who does not punish the active player. Otherwise, the rightmost player in the list who can be punished without punishing the active player is selected. The active player is then supposed to announce his favorite player for termination among the players that punish the selected scapegoat.

To formalize these ideas, define for $n \notin P^{*}$,

$$
P(n)=\left\{p \in P^{*} \mid(n, p) \text { is a safe step }\right\},
$$


and for $n, m \in N$, such that $(n, m)$ is not safe, define

$$
Q(n, m)=\left\{p \in P^{*} \mid p \text { punishes } n \text { and }(p=m \text { or } p \text { does not punish } m)\right\} \text {. }
$$

Notice that $P(n)$ and $Q(n, m)$ are nonempty under the given conditions.

For $n \in N$ and $p \in P^{*}$, such that $(n, p)$ is not safe, choose

$$
\bar{q}(n, p) \in \operatorname{argmax}\left\{v(q)_{p} \mid q \in Q(n, p)\right\} .
$$

Thus, $\bar{q}(n, p)$ represents a player that is most favorable for the persistent player $p$ to punish player $n$ with. Notice that $v(\bar{q}(n, p))_{p} \geq v(p)_{p}$.

For a list of players $\mathbf{s}=s_{1}-s_{2}-\cdots-s_{k}$ and $p \in P^{*}$, define

$$
q(\mathbf{s}, p)= \begin{cases}p & \text { if }\left(s_{i}, p\right) \text { is safe for all } i \in\{1, \ldots, k\} \\ \bar{q}\left(s_{j}, p\right) & \text { if }\left(s_{j}, p\right) \text { is not safe and }\left(s_{i}, p\right) \text { is safe for all } i \in\{j+1, \ldots, k\} .\end{cases}
$$

Notice that $q(-, p)=q(p, p)=p$ for all $p \in P^{*}$. Here, - denotes the empty list.

For a list $\mathbf{s}$ and $m \notin P^{*}$, choose

$$
p(\mathbf{s}, m) \in \operatorname{argmax}\left\{v(q(\mathbf{s}, p))_{m} \mid p \in P(m)\right\} .
$$

Thus, $p(\mathbf{s}, m)$ represents a best safe persistent player for $m \notin P^{*}$, under the assumption that play will terminate at $q(\mathbf{s}, p(\mathbf{s}, m))$. Notice that $v(q(\mathbf{s}, p(\mathbf{s}, m)))_{m}>v(m)_{m}$.

A description of subgame-perfect equilibrium play can now be given. Define a game situation as a pair $\langle\mathbf{s}, m\rangle$, where $\mathbf{s}$ is a list of players, and $m$ represents the active player of the game. End of game is indicated by $\langle-,-\rangle$. The rules distinguish between $m \in P^{*}$ and $m \notin P^{*}$. They tell the active player $m$ what he is supposed to do, and they describe how the game situation changes, depending on $m$ 's action.

R1 (a) Game situation: $<\mathbf{s}, m>$ with $m \in P^{*}$.

(b) Action:

i. If $m \neq q(\mathbf{s}, m)$ : Announce $q(\mathbf{s}, m)$.

ii. If $m=q(\mathbf{s}, m)$ : Quit.

(c) New game situation:

i. If $r=q(\mathbf{s}, m)$ is announced: $\langle-, r\rangle$.

ii. If $r \neq q(\mathbf{s}, m)$ is announced: $\langle m, r\rangle$.

iii. If the game is terminated: $\langle-,->$.

R2 (a) Game situation: $<\mathbf{s}, m>$ with $m \notin P^{*}$.

(b) Action: Announce $p(\mathbf{s}, m)$.

(c) New game situation:

i. If $r$ is announced such that $(m, r)$ is safe: $\langle\mathbf{s}, r\rangle$.

ii. If $r$ is announced such that $(m, r)$ is not safe: $\langle\mathbf{s}-m, r\rangle$.

iii. If the game is terminated: $\langle-,->$. 
Notice that everyone is either supposed to quit or to announce a persistent player. Therefore, if all players follow the rules, rule R2 will be applied at most once. Rule R1 will be applied at most twice: If the first application is an announcement, then the game situation becomes $\langle-, r\rangle$ with $r \in P^{*}$. Since $q(-, n)=n$ for all $n \in P^{*}$, player $r$ is supposed to quit at the next application of R1. Hence, the game always terminates after at most two announcements if players follow the rules.

Only one scapegoat player is necessary for the example of Section 2 in order to play a subgame-perfect equilibrium. To understand the use of lists, consider the following example with 10 players, given by the payoff matrix below, where the columns correspond to the payoff vectors $v(n), n=1, \ldots, 10$. The lowest possible payoff at termination is 1 . To highlight the other payoffs, the lowest payoff is denoted by '-' in the matrix.

\begin{tabular}{r|cccc|ccc|ccc|} 
& 1 & 2 & 3 & 4 & 5 & 6 & 7 & 8 & 9 & 10 \\
\hline 1 & - & - & - & - & 2 & 3 & 2 & - & 2 & 2 \\
2 & - & - & - & - & 2 & 3 & 2 & 2 & 2 & 2 \\
\hline 3 & - & - & - & - & - & 2 & 2 & 2 & 3 & 2 \\
4 & - & - & - & - & 2 & 2 & 2 & 2 & 3 & 2 \\
\hline 5 & - & - & - & - & 2 & - & 3 & - & - & - \\
6 & - & - & - & - & 3 & 2 & - & - & - & - \\
7 & - & - & - & - & - & 3 & 2 & - & - & - \\
\hline 8 & - & - & - & - & - & - & - & 2 & - & 3 \\
9 & - & - & - & - & - & - & - & 3 & 2 & - \\
10 & - & - & - & - & - & - & - & - & 3 & 2 \\
\hline
\end{tabular}

Notice that the persistent set for this example is $P^{*}=P^{1}=\{5,6,7,8,9,10\}$. For the non-persistent players 2 and 4, every step to a player in $P^{*}$ is safe. For player 1 , the steps to 5,6 , and 7 are safe, as well as the step to 10 . For player 3 , the steps to 8,9 , and 10 are safe, as well as the step to 7 .

We are interested in $p(3,1), p(1,2)$ and $p(3-1,2)$. To find $p(3,1)$, we first calculate $q(3, p)$ for $p \in P(1)=\{5,6,7,10\}$. We have $q(3, p)=p$ for $p=5,7,10$ and $q(3,6)=5$. Then $v(q(3, p))_{1}=2$ for all $p \in P(1)$. Therefore, we may choose $p(3,1) \in P(1)$ arbitrarily. We choose $p(3,1)=5$. To find $p(1,2)$, it is sufficient to observe that $q(1,6)=6$ and that 6 is the unique player where the payoff to player 2 is the maximum of 3 . Since $6 \in P(2)$, it follows that $p(1,2)=6$. To find $p(3-1,2)$, notice that $q(3-1,6)=\bar{q}(3,6)=5$. For $p \in P(2)$, $p \neq 6$, we have $q(3-1, p) \neq 6$, since 6 does not punish either of the two players on the list. It then follows that $v(q(3-1, p))_{2}=2$ for all $p \in P(2)$, so we can choose $p(3-1,2)$ arbitrarily in $P(2)=\{5,6,7,8,9,10\}$. We choose $p(3-1,2)=8$.

If the game situation is $\langle 3,1\rangle$, then player 1 is supposed to announce $p(3,1)=5$, and the game situation becomes $\langle 3,5\rangle$. Since $q(3,5)=5$, player 5 is now supposed to terminate. This yields 2 for player 1 . If the game situation is $\langle 1,2\rangle$, then player 2 is supposed to announce 6 after which the game situation becomes $<1,6>$. Since $q(1,6)=6$, player 6 is then supposed to terminate. This yields 3 for player 1 . We see that game situation $<1,2>$ leads to a better outcome for player 1 than game situation $<3,1\rangle$, if players follow rules $\mathrm{R} 1$ and $\mathrm{R} 2$.

If the game situation is $\langle 3,1>$ and player 1 announces player 2 , in violation of rule $\mathrm{R} 2$, then the new game situation becomes $<3-1,2>$. Assuming that the other players still 
follow the rules, player 2 will subsequently announce $p(3-1,2)=8$ and the game situation becomes $\langle 3-1,8\rangle$. Since $q(3-1,8)=\bar{q}(1,8)=8$, play will then terminate at 8 and player 1 will receive the minimal payoff of 1 . We see that player 1 is punished for his violation of the rules, as required. Notice however that this is because player 3 is still on the list. If player 1 had replaced 3 as the only scapegoat, then player 2 would have announced $p(1,2)=6$, and play would have terminated there, with the maximal payoff of 3 for player 1 . The use of lists makes this kind of manipulations impossible.

We claim that rules R1 and R2 constitute a subgame-perfect equilibrium for the general case. The following lemma is helpful.

Lemma 3 Let the game situation be $<\mathbf{s}, m>$, where $s \in P^{*}$ is the rightmost element of s. Suppose that all players, except possibly s, play according to rules $R 1$ and $R 2$. Then the game will terminate at $r \in P^{*}$ with $v(r)_{s} \leq v(s)_{s}$ or, after at least one announcement, it will reach a game situation $\langle\mathbf{t}, s>$ with $s$ as the rightmost element of $\mathbf{t}$ or with $\mathbf{t}=-$.

Proof. If $m \in P^{*} \backslash\{s\}$, then player $m$ will follow rule R1. Since $(s, m)$ is not safe, he will announce $\bar{q}(s, m)$, and the game situation becomes $<-, \bar{q}(s, m)>$. We are done if $s=\bar{q}(s, m)$. Otherwise, player $\bar{q}(s, m)$ will terminate, in which case the lemma follows from the fact that $\bar{q}(s, m)$ punishes $s$.

If $m \notin P^{*}$, then player $m$ will play according to R2. The game will therefore continue to the game situation $\langle\mathbf{s}, p(\mathbf{s}, m)>$. If $p(\mathbf{s}, m)=s$, we are done. If $p(\mathbf{s}, m) \neq s$, the lemma also follows, since we just proved the lemma for game situations $\langle\mathbf{s}, m\rangle$ with $m \in P^{*} \backslash\{s\}$.

If $m=s$ and $s$ quits, the lemma trivially follows. If $m=s$ and $s$ announces $s$, then the game situation becomes $\langle-, s\rangle$, since $s=\bar{q}(s, s)=q(\mathbf{s}, s)$. So also in this case the lemma follows. If $m=s$ and $s$ announces $m^{\prime} \neq s$, then $s$ did not announce $q(\mathbf{s}, s)$, so the game situation becomes $\left\langle s, m^{\prime}\right\rangle$. We already proved the lemma for such game situations.

We now prove our main result.

Theorem 4 The strategy profile determined by the rules $R 1$ and $R 2$ constitutes a pure subgame-perfect equilibrium.

Proof. We check, for both rules, that the active player cannot gain by deviating from the rule, assuming that all other players follow the rules.

R1. Let the game situation be $<\mathbf{s}, m>$ with $m \in P^{*}$. If player $m$ follows rule R1, the game will terminate at $q(\mathbf{s}, m)$, and player $m$ receives at least $v(m)_{m}$.

Suppose that $m$ decides to deviate from R1. We check, for the three possible types of deviation, that $m$ 's payoff will be at most $v(m)_{m}$.

i. If $m$ announces $r=q(\mathbf{s}, m)$, it is a deviation only if $q(\mathbf{s}, m)=m$. In that case, the game situation becomes $\langle-, m\rangle$ and player $m$ is again supposed to terminate. Player $m$ may decide to deviate again, but since Lemma 3 applies when he announces someone other than himself, play will either terminate at a player that punishes $m$, or reach again a game situation $\langle\mathbf{t}, m>$ with $\mathbf{t}=-$ or with $m$ as the rightmost element of t. Hence, $m$ 's payoff will be at most $v(m)_{m}$. 
ii. If $m$ announces $r \neq q(\mathbf{s}, m)$, then the new game situation will be $\langle m, r>$. By Lemma 3, the game will either terminate at a player that punishes $m$, or it will reach a game situation $\langle\mathbf{t}, m\rangle$ with $\mathbf{t}=-$ or with $m$ as the rightmost element of t. We have proved already that this game situation yields at most $v(m)_{m}$ for player $m$.

iii. If the game is terminated, player $m$ receives exactly $v(m)_{m}$.

R2. Let the game situation be $\left\langle\mathbf{s}, m>\right.$ with $m \notin P^{*}$. Player $m$ is supposed to announce $p(\mathbf{s}, m)$, and play will subsequently terminate at $q(\mathbf{s}, p(\mathbf{s}, m))$. Recall that $v(q(\mathbf{s}, p(\mathbf{s}, m)))_{m}>v(m)_{m}$. Suppose player $m$ deviates from R2.

i. If player $m$ announces $r$ such that $(m, r)$ is not safe, then the new game situation will be $\left\langle\mathbf{s}-m, r>\right.$. The step $(m, r)$ is not safe and $m \notin P^{*}$, which implies $r \neq m$, so $r$ will play according to the rules.

- If $r \in P^{*}$, player $r$ will play according to $\mathrm{R} 1$, and the game will eventually terminate at $q(\mathbf{s}-m, r)$. Since $(m, r)$ is not safe, it follows that $q(\mathbf{s}-m, r)=$ $\bar{q}(m, r) \in Q(m, r)$. Hence, termination is at a player that punishes $m$ : this is no improvement for $m$.

- If $r \notin P^{*}$, player $r$ will follow rule R2, and the game will eventually terminate at $q(\mathbf{s}-m, p(\mathbf{s}-m, r))$.

- If $(m, p(\mathbf{s}-m, r))$ is not safe, then $q(\mathbf{s}-m, p(\mathbf{s}-m, r))=\bar{q}(m, p(\mathbf{s}-m, r)) \in$ $Q(m, r)$, so then the game will terminate at a player that punishes $m$ : no improvement.

- If $(m, p(\mathbf{s}-m, r))$ is safe, then $q(\mathbf{s}-m, p(\mathbf{s}-m, r))=q(\mathbf{s}, p(\mathbf{s}-m, r))$. Since

$$
v(q(\mathbf{s}, p(\mathbf{s}-m, r)))_{m} \leq \max \left\{v(q(\mathbf{s}, p))_{m} \mid p \in P(m)\right\}=v(q(\mathbf{s}, p(\mathbf{s}, m)))_{m},
$$

it follows that this is no improvement for $m$ either.

ii. If $m$ announces $r$ such that $(m, r)$ is safe, then the new game situation will be $<\mathbf{s}, r>$.

- If $r \in P^{*}$, then $r \neq m$, so player $r$ will play according to R1. The game will subsequently terminate at $q(\mathbf{s}, r)$. This is no improvement for $m$, since

$$
v(q(\mathbf{s}, r))_{m} \leq \max \left\{v(q(\mathbf{s}, p))_{m} \mid p \in P(m)\right\}=v(q(\mathbf{s}, p(\mathbf{s}, m)))_{m} .
$$

- If $r \notin P^{*}$, it could be that $r=m$, in which case the game situation did not change. This will not lead to an improvement. If $r \neq m$, then $r$ will follow rule $\mathrm{R} 2$, and the game will terminate at $q(\mathbf{s}, p(\mathbf{s}, r))$. Notice that the step $(m, p(\mathbf{s}, r))$ is safe, by the fact that $(m, r)$ and $(r, p(\mathbf{s}, r))$ are both safe and by the transitivity of safe steps. Therefore,

$$
v(q(\mathbf{s}, p(\mathbf{s}, r)))_{m} \leq \max \left\{v(q(\mathbf{s}, p))_{m} \mid p \in P(m)\right\}=v(q(\mathbf{s}, p(\mathbf{s}, m)))_{m} .
$$

We see that this is no improvement for $m$ either. 
iii. Finally, if player $m$ quits, he receives $v(m)_{m}$ : no improvement.

The results in this article can easily be extended to games, where players control more than one state, provided that transitions remain free.

\section{References}

Bloch F., 1996, Sequential Formation of Coalitions in Games with Fixed Payoff Division. Games and Economic Behavior: 14, 90-123.

Fudenberg D. And Levine D., 1983, Subgame-Perfect Equilibria of Finite- and InfiniteHorizon Games. Journal of Economic Theory: 31, 251-268.

Liggett T.M. And Lippman S.A., 1969, Stochastic Games with Perfect Information and Time Average Payoff. SIAM Review: 11, 604-607.

Solan E. AND Vieille N., 2003, Deterministic multi-player Dynkin games. Journal of Mathematical Economics: 39, 911-929.

Solan E., 2005, Subgame-Perfection in Quitting Games with Perfect Information and Differential Equations. Mathematics of Operations Research: 30(1), 51-72.

Thuijsman F. And Raghavan T.E.S., 1996, Perfect Information Stochastic Games and Related Classes. International Journal of Game Theory: 26, 403-408. 